\title{
COEFICIENTES DOWNGRADE PARA SORTIMENTOS DE TORAS DE PINUS
}

\author{
DOWNGRADE COEFFICIENTS FOR ASSORTMENT PINUS LOGS \\ Edson Luis Serpe', Afonso Figueiredo Filho' ${ }^{2}$, Julio Eduardo Arce \\ ${ }^{1}$ LWARCEL, Lençóis Paulista, São Paulo, Brasil-serpe.edson@gmail.com \\ ${ }^{2}$ Universidade Estadual do Centro Oeste, Irati, Paraná, Brasil -afigfilho@gmail.com \\ ${ }^{3}$ Universidade Federal do Paraná, Curitiba, Paraná, Brasil - jarce@ufpr.br
}

RESUMO

\begin{abstract}
A determinação de volumes comerciais por sortimento é de grande importância para a valorização dos ativos florestais. $O$ objetivo deste estudo foi determinar coeficiente downgrade para sortimentos de toras de pinus e analisar como estes coeficientes podem contribuir para a maior assertividade do volume total e por sortimentos em florestas. Foram utilizadas 100 árvores-amostra, selecionadas proporcionalmente de acordo com a distribuição de frequência por classe diamétrica do censo florestal. A colheita florestal foi realizada de forma mecanizada, utilizando o sistema de árvores inteiras (Full tree). Os coeficientes downgrade foram estimados utilizando-se o percentual do volume estimado por sortimento, e o percentual do volume por sortimento real, sendo definidos os coeficientes de downgrade para sortimentos, com a divisão entre o volume real e volume estimado. Os efeitos econômicos das perdas por downgrade de sortimentos foram avaliados e apresentaram perdas na receita bruta de $5,41 \%$, reduzindo em $\mathrm{R} \$ 1.300,95 . \mathrm{ha}^{-1}$.
\end{abstract}

PALAVRAS-CHAVE: Colheita florestal, Desperdícios, Perdas, Qualidade, Reclassificação.

\section{ABSTRACT}

The determination of commercial volumes by assortment is of great importance for the valuation of the forest assets. The objective of this study was to determine downgrade coefficients for assortments of pine logs and to analyze how these coefficients can contribute to the greater assertiveness of the total volume and by assortments in forests. We used 100 sample trees, selected proportionally according to the frequency distribution by diameter class of the forest census. The forest harvest was carried out in a mechanized way, using the full tree system. The downgrade coefficients were estimated using the percentage of the estimated volume by assortment and the percentage of the volume by actual assortment, the downgrade coefficients for assortments being defined, with the division between the actual volume and the estimated volume. The economic effects of losses due to downgrade of assortments were evaluated and presented losses in gross revenue of $5.41 \%$, reducing by $R \$ 1,300.95$. ha ${ }^{-1}$.

KEY WORDS: Forest harvesting, Waste, Loss, Quality, Reclassification. 


\section{INTRODUÇÃO}

A atividade florestal possui respeitável papel na economia brasileira, estando inseridas neste ramo, reflorestadoras, empresas de processamento de madeira (celulose e papel, painéis de madeira reconstituída, siderurgia à carvão vegetal e industrial madeireiro especializado na laminação de toras e madeira serrada) e investidores (nacionais e internacionais) (IBÁ, 2015).

Por ser uma alternativa econômica relevante no cenário nacional, são realizadas muitas transações comerciais de ativos florestais (compra e venda de florestas), as quais demandam de informações para a estimativa volumétrica e principalmente a valoração do ativo em negociação.

Para a tomada de decisão dos investidores, a realização de um inventário florestal com qualidade é primordial. No entanto, apenas a estimativa do volume total, não deve ser considerada de forma isolada para a tomada de decisão.

Em ativos conduzidos para atender a demanda de multiprodutos (laminação, serraria e celulose), existe a grande importância em mensurar os sortimentos, pois possuem diferentes valores de mercado.

Para a tomada de decisão, além das informações quantitativas, um fato muitas vezes ignorado são as perdas ocasionadas pela atividade da colheita florestal, que podem ser maiores ou menores dependendo da qualidade da floresta e da operação da colheita florestal.

A estimativa do volume por classes de sortimentos, pressupõe que não existem defeitos nas árvores e toda a madeira é comercializável. No entanto, em qualquer floresta existem particularidades do fuste, que podem ser consideradas para estimativa do volume por classe de sortimento mais próximo do real (FOREST SERVICE, 2007).

Fatores biológicos (bifurcação e retidão do fuste), danos causados por fatores bióticos (macaco-prego), abióticos (vento) ou por fatores mecânicos (máquinas florestais), podem proporcionar redução no volume de madeira comercializável e/ou downgrade de sortimentos e consequentemente perdas econômicas (FOREST SERVICE, 2007).

O termo "downgrade" foi utilizado por Mello (2004), em pesquisa de utilização da madeira sobressalente do sortimento do torno grande para torno pequeno ou para serraria e do sortimento do torno pequeno para serraria.

McCurdy et al. (2005), em pesquisa de determinação da cor em tábuas de Pinus radiata D. Don, verificaram que os defeitos de descoloração reduzem o valor dos produtos, resultando em perdas relevantes de madeira. Isso também induz ao downgrade, ou seja, que o produto seja rebaixado ou reclassificado como resíduo.

Hébert et al. (2016) citaram que a quantidade e tamanho dos nós podem ocasionar o downgrade, rebaixando a qualidade da madeira para uso na construção civil.

Nieuwenhuis e Fitzpatrick (2002) verificaram o downgrade do volume em toras provocado por tempestade em florestas, quantificando as perdas de volume e receita, ocasionados pelo downgrade de sortimentos.

O objetivo deste trabalho foi propor coeficientes de downgrade para sortimentos de toras acarretadas por perdas de colheita e qualidade da floresta, os quais podem contribuir para a maior assertividade do volume total e por sortimentos em inventários florestais.

\section{MATERIAL E MÉTODOS}

\section{Localização da área de estudo}

A área de estudo localiza-se na fazenda Mato Queimado I, município de Bituruna, região sul do estado do Paraná, nas coordenadas UTM (Universal Transversa de Mercator) 431.383,731 m E e 7.104.262,170 m N (Datum Sirgas 2000, Zona 22J), em uma altitude média de 1.216 m.s.n.m, pertencente a empresa Remasa Reflorestadora S.A.

\section{Coleta dos dados}

Os dados foram coletados em uma área de 43,579 hectares de plantio misto de Pinus elliottii Engelm e Pinus taeda L. implantado em relevo suave ondulado. A implantação deste povoamento foi realizada no ano de 1998, com espaçamento de $3 \mathrm{~m}$ x $3 \mathrm{~m}$ (1.111 árvores.ha ${ }^{-1}$ ).

O mesmo foi conduzido com manejo clearwood, sendo realizado o primeiro desbaste seletivo baixo (colheita de árvores suprimidas ou com problemas de qualidade como: tortuosidades, bifurcadas ou com danos causados pelo ataque de pragas).

O primeiro desbaste foi realizado em 2007 (9 anos de idade, remanescendo 667 árvores.ha $\left.{ }^{-1}\right), \quad$ o segundo desbaste seletivo em 2010 (12 anos de idade, remanescendo 440 árvores.ha-1) e o corte raso a partir de setembro de 2014 (aos 16 anos de idade). Além disso, foram realizadas podas até $6 \mathrm{~m}$ de altura.

Nesta pesquisa, foi realizado o inventário a $100 \%$ (censo florestal), quando o plantio tinha 16 anos de idade, sendo mensurados o diâmetro a $1,3 \mathrm{~m}$ do solo (DAP), com a utilização de uma suta, e a altura total mensuradas com 
o hipsômetro Vertex, de todas as 19.329 árvores.

Com os dados de inventário florestal, foram selecionadas 100 árvores-amostra, conforme a distribuição de frequência por classe diamétrica encontrada.

O número de árvores derrubadas por classe diamétrica foi definido proporcionalmente à frequência encontrada no inventário florestal.

A colheita florestal foi realizada de forma mecanizada, utilizando o sistema de árvores inteiras (Full tree). As árvores foram derrubadas utilizando-se de escavadeira equipada com cabeçote Feller direcional com corrente de passo $3 / 4$ " (espessura do filete de serragem de 1,5 cm) e dispostas de forma a facilitar a coleta dos dados (Figura 1).

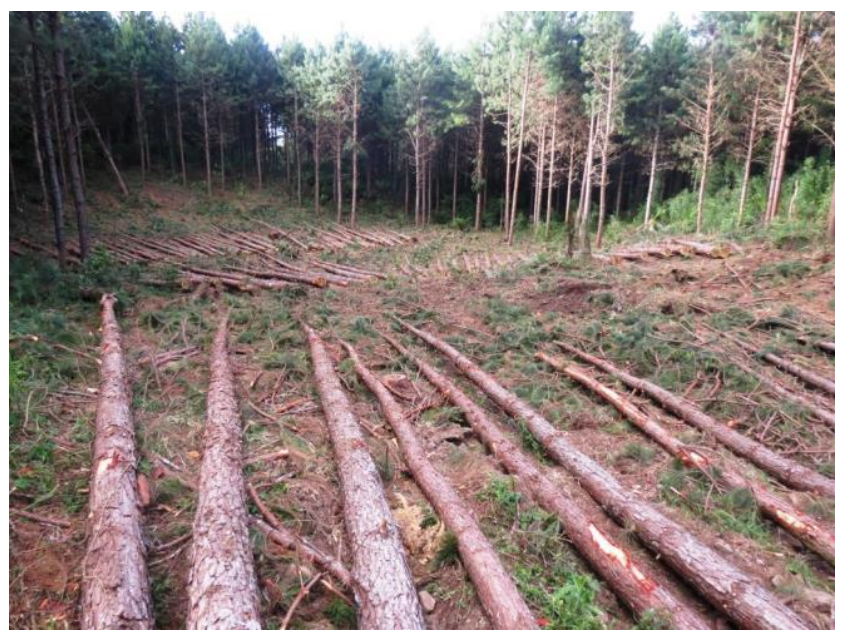

Figura 1. Disposição dos fustes para cubagem.

Logo após o corte, foi realizada a medição da altura do toco com trena retrátil. Antes do traçamento "seccionamento" dos fustes em toras, foi realizada a coleta de dados de cubagem utilizando o método de Smalian (medição dos diâmetros da ponta grossa e ponta fina e comprimento da tora) de acordo com os sortimentos praticados pela empresa (Tabela 1).

Tabela 1. Sortimentos produzidos pela empresa.

\begin{tabular}{ccc}
\hline Classe & Classe de diâmetro $(\mathbf{c m})$ & Comprimento $(\mathbf{m})$ \\
\hline Serraria 1 & $\geq 23$ & 2,65 \\
Serraria 2 & $\geq 16$ & 2,65 \\
Celulose & $\geq 8$ & 1,80 \\
\hline
\end{tabular}

Após a coleta dos dados de cubagem, os fustes foram extraídos por arraste com Skidder até a borda do talhão para serem seccionados.

Os fustes foram seccionados e separados por classe de diâmetro, conforme Tabela 1, por escavadeira hidráulica com cabeçote harvester com sabre e corrente com passo 0,404 ". A Figura 2 apresenta as pilhas de toras, separadas de acordo com o sortimento.

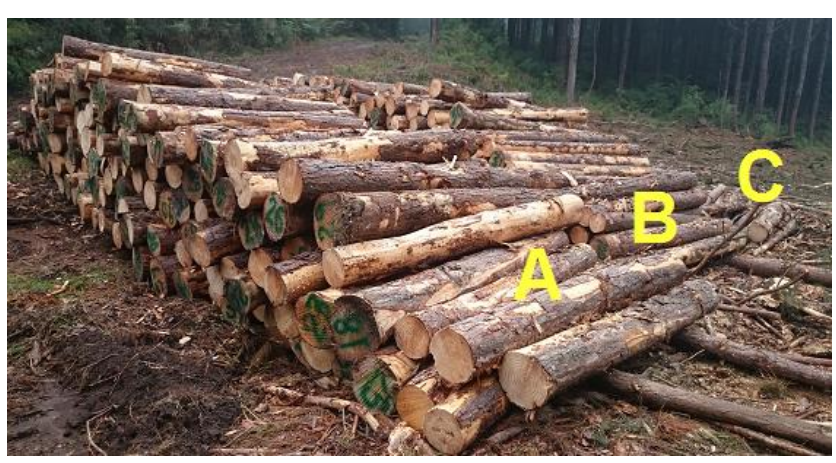

Figura 2. Disposição das pilhas de toras por sortimento: A $=$ Serraria $1, B=$ Serraria 2 e C = Celulose.

Após o seccionamento, as toras geradas para cada classe de diâmetro, foram medidas individualmente pelo método de Smalian (medição dos diâmetros da ponta grossa e ponta fina e comprimento da tora).

\section{Determinação dos volumes}

Para a determinação dos volumes por sortimento, foi utilizado o método de cubagem de Smallian, conforme equação:

$$
\mathrm{Vt}=\frac{\left(\frac{\pi(\mathrm{dpf})^{2}}{4}+\frac{\pi(\mathrm{dpg})^{2}}{4}\right)}{2} \times \mathrm{L}
$$

Em que: $\mathrm{Vt}=$ Volume da tora $\left(\mathrm{m}^{3}\right) ; \mathrm{dpf}=$ diâmetro da tora na ponta fina $(\mathrm{m})$; $\mathrm{dpg}=$ diâmetro da tora na ponta grossa (m); e L = comprimento da tora.

O volume estimado, considera os volumes obtidos da cubagem antes do processamento. Entretanto, o volume real é considerado após o seccionamento e seleção das toras por sortimento.

\section{Downgrade de sortimentos e suas perdas econômicas}

As estimativas de sortimentos produzidas por funções de afilamento não levam em consideração o fato de que as árvores do povoamento não são perfeitas. Apesar do elevado padrão de melhoramento genético existente para as florestas de pinus, as árvores podem apresentar defeitos que favorecem o downgrade de um sortimento para outro.

O downgrade de um sortimento é definido como o rebaixamento, reclassificação ou migração de uma tora com diâmetro superior "Serraria $1 \geq 23 \mathrm{~cm}$ " para a classe de sortimento com toras de diâmetro inferior "Serraria $2 \geq$ 16 cm", reduzindo seu potencial econômico. Isto pode 
ocorrer devido fatores biológicos, como: tortuosidades e bifurcações do tronco, fatores bióticos, como: danos no tronco por pragas (danos por macaco-prego), dentre outros (Figura 3).

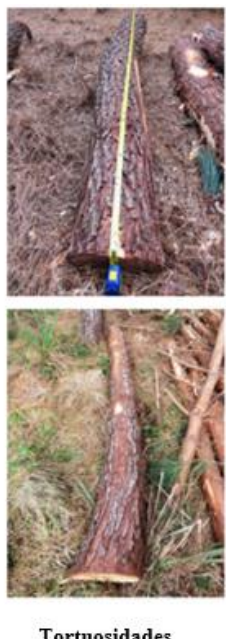

(A)
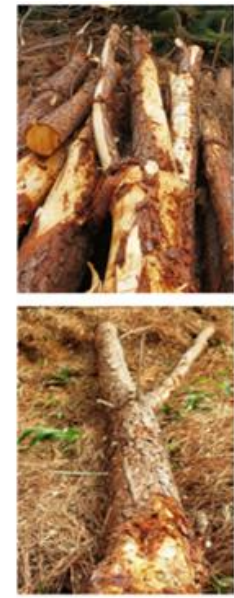

Bifurcações

(B)
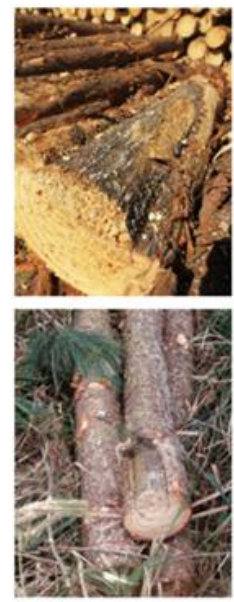

Danos por macaco-prego (C)
Figura 3. Downgrade Serraria $1 /$ Serraria 2 (A), Downgrade Serraria 2/Celulose (B), Downgrade Serraria 2/Celulose (C).

Além desses fatores, o downgrade de sortimentos pode ainda ser resultado de fatores mecânicos da colheita florestal, devido à quebra do fuste, imprecisão dos cabeçotes processadores que podem gerar erros no diâmetro e comprimento das toras.

$\mathrm{Na}$ literatura, vários artigos estão disponíveis para estimar os sortimentos em classes de diâmetros e comprimentos. Porém, são raros os trabalhos que estudaram o downgrade de sortimento de uma classe economicamente maior para uma menor.

Os coeficientes de downgrade para sortimento são calculados pela divisão entre o percentual de volume real por sortimento e percentual de volume estimado por sortimento, conforme equação:

$$
\text { Dgs }=\frac{\% \mathrm{VR}}{\% \mathrm{VE}}
$$

Em que: Dgs = Coeficiente downgrade para sortimento; $\% \mathrm{VR}=$ percentual de volume real por sortimento; $\mathrm{e} \% \mathrm{VE}=$ percentual de volume estimado por sortimento.

As perdas econômicas geradas pelo downgrade de sortimentos na colheita florestal, foram calculadas pela multiplicação dos volumes estimados e volumes reais por sortimento, pelos preços da madeira praticados pela empresa no ano de 2016 (Tabela 2).
Tabela 2. Preço de toras praticados na região de União da Vitória, estado do Paraná, 2016.

\begin{tabular}{ccc}
\hline Classe & Classe de diâmetro $\mathbf{( c m )}$ & $\mathbf{R} \mathbf{\$} / \mathbf{m}^{\mathbf{3}}$ \\
\hline Serraria 1 & $\geq 23$ & 122,40 \\
Serraria 2 & $\geq 16$ & 73,69 \\
Celulose & $\geq 8$ & 32,53 \\
\hline
\end{tabular}

\section{RESULTADOS E DISCUSSÃO}

Os percentuais de volume por sortimentos estimados são diferentes dos percentuais encontrados após o processamento, caracterizando a existência de downgrade provocado pela colheita florestal e pela qualidade das árvores.

A presente pesquisa indica que o downgrade de sortimentos em uma floresta de Pinus de dezesseis anos em regime de manejo com desbaste, deve considerar os coeficientes de downgrade por sortimento, buscando gerar informações mais próximas dos volumes que realmente são comercializáveis.

Assim, os sortimentos com casca de cada classe de sortimentos, encontrados nos inventários florestais, deveriam ser multiplicados por coeficientes downgrade como os apresentados na Tabela 3.

Tabela 3. Coeficientes "downgrade" para sortimentos.

\begin{tabular}{cccc}
\hline \multirow{2}{*}{ Classe } & \multicolumn{2}{c}{ \% Sortimento } & \multirow{2}{*}{ Dgs } \\
\cline { 2 - 3 } & $\begin{array}{c}\text { Volume } \\
\text { Estimado }\end{array}$ & $\begin{array}{c}\text { Volume } \\
\text { Real }\end{array}$ & \\
\hline Serraria 1 & 58,51 & 49,34 & 0,8433 \\
Serraria 2 & 32,36 & 39,44 & 1,2188 \\
Celulose & 9,13 & 11,22 & 1,2289 \\
\hline
\end{tabular}

Serraria 1: $\geq 23 \mathrm{~cm}$; Serraria 2: $\geq 16 \mathrm{~cm}$; Celulose: $\geq 8 \mathrm{~cm}$. Dgs: Coeficiente downgrade para sortimento.

Verifica-se o maior downgrade no volume dos sortimentos "Serraria 1" para os sortimentos "Serraria 2" e "Celulose", sendo possível concluir que tal fato é decorrente de fatores biológicos como, tortuosidades e bifurcações do tronco; fatores bióticos tais como, danos no tronco por pragas (macaco-prego); fatores mecânicos da colheita florestal, devido à quebra do fuste, imprecisão dos cabeçotes processadores (erros no diâmetro e comprimento das toras) além de fatores relacionados a operação (falta de treinamento para o operador, cansaço físico ao final do turno por "movimento repetitivo" e dificuldade de operação no horário noturno).

As pesquisas de Campos e Tavares (2010) e Mendonça (2006), demonstraram o downgrade de sortimento 
quando comparado o volume estimado e o volume comercializado, e tal fato foi observado nesta pesquisa, produzindo subsídios para a utilização de coeficientes downgrade para sortimentos.

Mendonça (2006), em avaliação de uma metodologia para otimização do volume de toras comerciais de Eucalyptus spp. em função da qualidade do fuste, comparou o volume estimado pelo inventário e volume obtido na colheita, verificando o downgrade de sortimentos das classes de toras.

Campos e Tavares (2010), em pesquisa da influência do comprimento e otimização de toras em Pinus pinaster Aiton, verificaram o downgrade de sortimentos considerando os volumes por sortimento otimizados e os volumes colhidos.

Considerando que o downgrade de sortimentos pode ocorrer por falhas na colheita florestal, seja ela semimecanizada ou mecanizada, os autores Rosa e Oliveira (2014), em análise da qualidade do processamento de madeira em dois sistemas de colheita florestal (full tree e cut to lenght) de plantios de Pinus utilizando três classes de sortimento, verificaram que, de modo geral, o processamento com cabeçote processador não apresentou precisão para as variáveis diâmetro e comprimento, quando comparados com a programação no computador de bordo. Os erros correspondem a problemas operacionais em função de defeitos na madeira (como galhos grossos, bifurcações e tortuosidades), além de problemas de ordem mecânica (aferição do cabeçote) e relacionados à forma de operar a máquina (falta de treinamento do operador).

Segundo os mesmos autores, no sistema de colheita full tree, $10 \%$ dos diâmetros e $30 \%$ dos comprimentos encontravam-se em não conformidade, em comparação com os valores reais e valores definidos no computador de bordo da máquina. Em relação à margem de tolerância para diâmetro, o percentual se manteve, já para o comprimento houve uma redução para $23 \%$. Para o sistema de colheita cut to lenght, o percentual de não conformidade para o diâmetro foi de $8 \%$ e para comprimento foi de $27 \%$. Considerando a margem de tolerância, o percentual dos diâmetros não se alterou, já para o comprimento foi reduzido para $3 \%$.

A pesquisa sobre a avaliação da qualidade operacional em cinco subsistemas de colheita florestal apontou o percentual de toras com comprimento acima da especificação entre 7,0 a 26,4\% e com comprimento abaixo da especificação entre de 8,0 a 25,6\% (JACOVINE et al., 2005).

Fiedler et al. (2013), em análise da qualidade da colheita florestal de eucalipto em diferentes declividades, verificaram que os comprimentos das toras fora do padrão determinado esteve entre 20,42 a $35,80 \%$.

As perdas econômicas considerando o downgrade de sortimentos na atividade de colheita florestal são apresentadas na Tabela 4.

Tabela 4. Efeitos econômicos das perdas por downgrade de sortimentos.

\begin{tabular}{cccc}
\hline \multirow{2}{*}{ Classe } & \multicolumn{2}{c}{ Receita bruta $\left(\mathbf{R} \mathbf{\text { .h }} \mathbf{h a}^{-\mathbf{1}}\right.$ ) } & \multirow{2}{*}{$\begin{array}{c}\text { Downgrade } \\
\text { (R\$.ha-1) }\end{array}$} \\
\cline { 2 - 3 } & Estimada & Real & \\
\hline Serraria 1 & $17.490,12$ & $14.748,97$ & $-2.741,15$ \\
Serraria 2 & $5.823,69$ & $7.097,85$ & $1.274,16$ \\
Celulose & 725,33 & 891,37 & 166,04 \\
Total & $\mathbf{2 4 . 0 3 9 , 1 4}$ & $\mathbf{2 2 . 7 3 8 , 1 9}$ & $\mathbf{- 1 . 3 0 0 , 9 5}$ \\
\hline
\end{tabular}

Serraria 1: $\geq 23 \mathrm{~cm}$; Serraria 2: $\geq 16 \mathrm{~cm}$; Celulose: $\geq 8 \mathrm{~cm}$.

O downgrade de sortimentos impactou diretamente na receita do sortimento "Serraria 1", o qual apresentou redução de 15,67\%. Com a reclassificação, ocorreu a redução da receita bruta em $\mathrm{R} \$ 1.300,95$ ha $^{-1}(5,41 \%)$.

\section{CONCLUSÕES}

As perdas econômicas devido ao downgrade de sortimentos, relacionados a fatores biológicos (tortuosidades e bifurcações), fatores bióticos (danos causados por macaco-prego) e fatores mecânicos da colheita florestal (imprecisão do cabeçote processador ou falha de operação do equipamento), impactaram expressivamente na receita do ativo florestal.

Os coeficientes downgrade para sortimentos são uma opção para a redução nas diferenças estimadas do volume comercializável por classe de sortimento, podendo contribuir para a maior assertividade dos inventários florestais.

Os volumes de sortimento estimados por funções de afilamento, levam em consideração que as árvores do povoamento são perfeitas, e que não existem perdas de colheita florestal, acarretando superestimativas de sortimentos em classes maiores e estimando receitas brutas inexistentes.

Ferramentas de gestão da qualidade para análise de defeitos ou não conformidades (diagrama de Ishikawa, diagrama de Pareto, 5W2H, PDCA e 6 Sigma), ferramentas de análise do modo e efeitos de falhas (FMEA) e ferramentas de análise do resultado (FCA), podem ser implementadas buscando a minimização de perdas. 


\section{AGRADECIMENTOS}

À Remasa Reflorestadora S.A., pela autorização e apoio no trabalho de campo.

\section{REFERÊNCIAS}

CAMPOS, C.; TAVARES, M. Influência do comprimento e origem do toro na optimização do aproveitamento e do valor de rolos de pinho bravo. Silva Lusitana, v.18, n.1, p.123-132, 2010.

FIEDLER, N.C. et al. Análise da qualidade da colheita florestal de eucalipto em diferentes declividades. Revista Científica Eletrônica de Engenharia Florestal, v.22, n.1, p.1-8, 2013.

FOREST SERVICE. National Forest Inventory - Republic of Ireland - Methodology, 2007. Department of Agriculture Fisheries and Food. Disponível em: https://www.agriculture.gov.ie/media/ migration/forestry/nationalforestinventory/nationalforestinvent orypublications/4350NFIMethodology.pdf

HÉBERT, F. et al. Effect of Tree Spacing on Tree Level Volume Growth, Morphology, and Wood Properties in a 25 -Year-Old Pinus banksiana Plantation in the Boreal Forest of Quebec. Forests, v.7, n. 276, p.1-16, 2016.

IBÁ. Relatório anual da IBÁ, ano 2015, ano base 2014, 2015. Disponível em: http://www.iba.org/images/shared/iba_2015.pdf

JACOVINE, L.A. et al. Avaliação da qualidade operacional em cinco subsistemas de colheita florestal, Árvore, v.29, n.3, p.391-400, 2005.

MCCURDY, M. et al. Measurement of colour development in Pinus radiata sapwood boards during drying at various schedules. Wood Technology. Maderas, v.7, n.2, p.79-85, 2005.

MELLO, A.A. Planejamento de uma empresa florestal considerando a manutenção do estoque de carbono. 2004. 115p. (Tese de doutorado).

MENDONÇA, A.R. Avaliação de uma metodologia para otimização do volume de toras comerciais de Eucalyptus sp. em função da qualidade do fuste. 2006. 90p. (Dissertação de mestrado).

NIEUWENHUIS, M; FITZPATRICK, P.J. An assessment of stem breakage and the reduction in timber volume and value recovery resulting from a catastrophic storm: an Irish case study. Forestry, v.75, n.5, p.513-523, 2002.

ROSA, M.O.; OLIVEIRA, F.M. Análise da qualidade do processamento de madeira em dois sistemas mecanizados de colheita florestal. Revista da União Latino-americana de Tecnologia, n.2, p.19-37, 2014. 